\title{
Planeamento da gravidez na adaptação à transição para a maternidade de grávidas infectadas pelo $\mathrm{VIH}$
}

Marco Pereira, * Maria Cristina Canavarro**

\section{RESUMO}

Objectivos: Analisar a influência do planeamento da gravidez na adaptação à transição para a maternidade de grávidas infectadas pelo vírus da imunodeficiência humana $(\mathrm{VIH})$, comparativamente a grávidas sem condição médica associada.

Tipo de estudo: Observacional, transversal, analítico, com avaliações em dois momentos: segundo trimestre de gravidez e dois a quatro dias após o parto.

Local: Hospitais da Universidade de Coimbra: Área de Gestão Integrada de Saúde Materno-Fetal - Unidade de Intervenção Psicológica da Maternidade Doutor Daniel de Matos; Maternidade Doutor Alfredo da Costa (Lisboa).

População: Noventa e oito mulheres: 47 grávidas seropositivas para o VIH e 51 grávidas sem condição médica de risco associada. Métodos: A adaptação à transição para a maternidade foi determinada pela aplicação às grávidas de três instrumentos de auto-preenchimento avaliando a sintomatologia psicopatológica (Brief Symptom Inventory), a reactividade emocional (Emotional Assessment Scale) e a qualidade de vida (WHOQOL-Bref). Foi analisada a associação entre a adaptação à transição para a maternidade e o planeamento, ou não, da gravidez, comparando-se esta análise em dois grupos: um de grávidas seropositivas para - VIH e outro de grávidas sem condição médica associada. Na análise foram usados métodos de estatística inferencial, sendo adoptado um nível de significância de 0,05.

Resultados: Os resultados obtidos apoiam a hipótese de que a gravidez não planeada se encontra associada a maiores dificuldades de adaptação na transição para a maternidade e, de forma mais acentuada, entre as mulheres infectadas pelo VIH. No pós-parto, a ausência de planeamento da gravidez mostrou-se significativamente associada a maior sintomatologia psicopatológica, maior reactividade emocional negativa e menor qualidade de vida.

Conclusões: Os resultados sublinham a importância de considerar o planeamento da gravidez na adaptação à gravidez e, sobretudo, ao pós-parto. Por conseguinte, reforçam também a importância de, por rotina, discutir os planos reprodutivos com as mulheres infectadas, previamente à decisão reprodutiva.

Palavras-chave: Planeamento familiar; Adaptação, Psicológica; VIH

\section{INTRODUÇÃO}

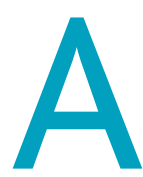

transição para a maternidade/parentalidade constitui-se como um importante marco na vida de uma mulher, bem como de um casal ou família, desafiando equilíbrios previamente estabelecidos ${ }^{1}$ e comportando múltiplas exi-

*Investigador de Pós-Doutoramento do Instituto de Psicologia Cognitiva, Desenvolvimento Vocacional e Social da Universidade de Coimbra, Faculdade de Psicologia e de Ciências da Educação da Universidade de Coimbra

**Professora Catedrática da Faculdade de Psicologia e de Ciências da Educação da Universidade de Coimbra, Instituto de Psicologia Cognitiva, Desenvolvimento Vocacional e Social gências para as novas mães, particularmente quando a gravidez não é planeada. ${ }^{2}$

A informação relativa à intenção de engravidar tem sido usada essencialmente para estimar a prevalência das gravidezes não planeadas, com o objectivo de implementar medidas de planeamento familiar. ${ }^{3}$ Estas gravidezes demonstraram estar associadas a custos individuais e sociais, ${ }^{4}$ assim como a efeitos no comportamento da mãe durante a gravidez, no resultado da gravidez e no desenvolvimento do bebé. ${ }^{5-6}$ Assumindo que a percepção que uma mulher tem da sua gravidez é um dos 
mais importantes factores com implicações na sua saúde e bem-estar, ${ }^{7}$ alguns autores têm associado o planeamento da gravidez à adaptação materna à gravidez ${ }^{8} \mathrm{e}$ ao puerpério. ${ }^{9-11}$ De facto, a evidência demonstra que as mulheres que não planearam a gravidez têm taxas mais elevadas de depressão, ansiedade e outros quadros psicopatológicos, ${ }^{8,12-15}$ havendo embora estudos que não encontraram qualquer associação $0^{16}$ ou que apresentaram resultados inconclusivos. ${ }^{17}$ Os estudos, no entanto, têm sido principalmente conduzidos em populações saudáveis, sendo escassos os estudos que associam a variável infecção por vírus da imunodeficiência humana (VIH) à adaptação psicológica na transição para a maternidade. ${ }^{16}$

No contexto da infecção VIH, o planeamento da gravidez, enquadrado no processo de tomada de decisão reprodutiva mais alargado, tem sido bastante estudado, ainda que essencialmente no contexto biomédico e, em concreto, reportando-se à decisão de prosseguimento ou interrupção da gravidez. Do ponto de vista psicossocial, uma vasta gama de estudos tem-se centrado na constelação de factores determinantes na decisão de engravidar ou não engravidar ou de prosseguir ou interromper a gravidez, ${ }^{18-22}$ embora poucos tenham avaliado a forma como esta decisão influencia a adaptação na transição para a maternidade.

Os resultados de um estudo nacional exploratório conduzido por Pereira e Canavarro ${ }^{23}$ sugerem uma influência significativa do planeamento da gravidez na adaptação ao pós-parto das mães seropositivas, na dimensão psicopatológica da depressão e nas emoções de ansiedade, culpa e tristeza. Concretamente, são as mulheres cuja gravidez não foi planeada que apresentam valores mais elevados nestes indicadores. Mais recentemente, um estudo conduzido no Canadá, ${ }^{24} \mathrm{com}$ o objectivo de analisar a prevalência e correlatos das gravidezes não planeadas em mulheres infectadas peloVIH, mostrou que estas se encontravam menos felizes quando a gravidez não tinha sido planeada.

Face ao exposto, o objectivo deste estudo consistiu em avaliar a influência do planeamento da gravidez na adaptação individual na transição para a maternidade em grávidas infectadas pelo VIH, comparativamente a grávidas sem patologia médica associada. No presente estudo estabelecemos como hipótese que as mulheres que não planearam a gravidez (quer seropositivas, quer sem risco médico associado) tenderão a manifestar maiores dificuldades de adaptação durante o período da gravidez. Colocámos ainda a hipótese de que a associação entre a ausência de planeamento da gravidez e as dificuldades de adaptação seria mais forte nos dias subsequentes ao parto.

\section{MÉTODOS}

O desenho do estudo foi observacional, transversal e analítico. O recrutamento das participantes foi realizado entre Abril de 2003 e Maio de 2008, nos serviços de Obstetrícia da Maternidade Doutor Daniel de Matos - Área de Gestão Integrada de Saúde Materno-Fetal dos Hospitais da Universidade de Coimbra e na Maternidade Doutor Alfredo da Costa (Lisboa). Foi usada uma técnica de amostragem não probabilística, por conveniência: as grávidas VIH convidadas a participar foram todas as inscritas na Consulta de Obstetrícia-Infecciosas; as grávidas sem VIH foram consecutivamente recrutadas enquanto aguardavam pela consulta de Obstetrícia na Maternidade.

As grávidas infectadas pelo VIH que constituem a amostra recrutada na Maternidade Doutor Daniel de Matos foram avaliadas individualmente pelo primeiro autor do presente estudo. O recrutamento da amostra na Maternidade Alfredo da Costa foi efectuada por duas psicólogas clínicas do Departamento de Psicologia da mesma maternidade e as grávidas sem VIH foram avaliadas por duas psicólogas da Unidade de Intervenção Psicológica da Maternidade Doutor Daniel de Matos. A recolha de dados em cada local obedeceu rigorosamente aos mesmos procedimentos.

Para a recolha de dados, foi feito previamente um pedido de colaboração voluntária no estudo, explicada a natureza e os objectivos do mesmo, garantida a confidencialidade e o anonimato das respostas aos questionários e assinado o consentimento informado, previamente aprovado pela Comissão de Ética do Conselho de Administração dos Hospitais da Universidade de Coimbra e pela Comissão de Ética da Maternidade Doutor Alfredo da Costa.

A recolha de dados foi realizada com base em duas entrevistas pessoais: uma no $2 .^{\circ}$ trimestre de gravidez e outra dois a quatro dias após o parto. Da primeira entrevista faziam parte uma ficha de dados sociodemográficos, duas grelhas de informação clínica e três questionários de auto-preenchimento relativos aos indica- 
dores de adaptação considerados. Da segunda entrevista fazia parte uma grelha de informação clínica sendo os três questionários relativos aos indicadores de adaptação aplicados uma segunda vez.

Relativamente às grelhas de informação clínica, uma primeira, de natureza obstétrica, incidia sobre os antecedentes obstétricos e a actual gravidez; uma segunda, aplicada apenas às grávidas VIH, dizia respeito à história médica da infecção por VIH, compreendendo os seguintes dados: duração da infecção; tempo de conhecimento; contexto de realização do testeVIH; categoria de transmissão; condição serológica do companheiro; e, no caso de ter filhos anteriores à actual gravidez, dados sobre o conhecimento da infecção no momento em que engravidou e a situação médica actual do(s) filho(s). A ficha clínica usada no segundo momento de avaliação incluía dados relativos ao parto e ao recém-nascido.

Os questionários usados como indicadores de adaptação foram o Brief Symptom Inventory, o Emotional Assessment Scale e o World Health Organization Quality of Life. O Brief Symptom Inventory (BSI) é um inventário de avaliação de sintomas psicopatológicos, ${ }^{25}$ no qual o indivíduo classifica o grau em que cada problema o afectou durante a última semana. O BSI avalia sintomatologia psicopatológica em termos de nove dimensões básicas de psicopatologia (Somatização; Obsessões-compulsões; Sensibilidade interpessoal; Depressão; Ansiedade; Hostilidade; Ansiedade fóbica; Ideação paranóide; e Psicoticismo) e três índices globais (Índice Geral de Sintomas; Total de Sintomas Positivos; e Îndice de Sintomas Positivos). Este último índice é considerado por Canavarro ${ }^{26}$ como o melhor discriminador entre indivíduos da população geral e aqueles que apresentam perturbações emocionais.

A Emotional Assessment Scale (EAS) tem como objectivo medir a reactividade emocional. ${ }^{27-28}$ A EAS é constituída por 24 itens, que correspondem a descrições de emoções consideradas fundamentais (Medo, Felicidade, Ansiedade, Culpa, Cólera, Surpresa e Tristeza), sendo especialmente útil na medida de níveis momentâneos e de mudança de emoções.

O World Health Organization Quality of Life, versão abreviada (WHOQOL-Bref) é um instrumento de avaliação da qualidade de vida, ${ }^{29-30}$ composto por 26 itens e organizado em quatro domínios: Físico, Psicológico, Relações sociais e Ambiente.
$\mathrm{Na}$ análise estatística dos dados foi utilizado o programa estatístico SPSS (Statistical Package for the Social Sciences - versão 17.0). Numa primeira fase, para a caracterização sociodemográfica da amostra e dos diferentes grupos que a compõem recorremos à estatística descritiva, incluindo frequências relativas, médias $(M)$ e desvios-padrão (DP). Com o objectivo de averiguar a existência de diferenças entre os dois grupos de estudo relativamente aos indicadores de adaptação mencionados, recorremos à estatística inferencial. Neste sentido, e quando as comparações se baseavam nas nove dimensões do BSI, nas sete emoções da EAS ou nos quatro domínios doWHOQOL-Bref, recorremos ao procedimento de análise multivariada da variância (MANOVA), concretamente à $2 \mathrm{X} 2 \mathrm{MANOVA}$, em que as variáveis independentes foram a infecção VIH e o planeamento de gravidez (planeada versus não planeada). Para análise da associação entre o planeamento de gravidez (variável dicotómica e discreta) e os indicadores de adaptação no pós-parto recorremos à correlação point biserial. Os testes estatísticos com probabilidades inferiores a 0,05 foram considerados estatisticamente significativos. A magnitude dos efeitos foi analisada através do $d$ de Cohen e o $V$ de Cramer, adoptando as convenções seguintes: efeito pequeno: $d$ de Cohen $\geq 0,20, V$ de Cramer $\geq 0,01$; efeito médio: $d$ de Cohen $\geq 0,50, V$ de Cramer $\geq 0,03$; efeito grande: $d$ de Cohen $\geq 0,80$, Vde Cramer $\geq 0,05)$. ${ }^{31}$

\section{RESULTADOS}

Um total de 105 grávidas (52 infectadas pelo VIH e 53 cuja gravidez não tinha associada qualquer condição médica) foi inicialmente contactado e todas aceitaram participar. Cinco grávidas VIH optaram pela interrupção da gravidez, 47 preencheram o protocolo de avaliação no $2 .^{\circ}$ trimestre de gravidez e, destas, 31 participaram na avaliação após o parto. Neste grupo com VIH, a razão mais frequente para a não participação na avaliação após o parto foi a realização do parto numa outra Maternidade. Do total de contactadas, 51 grávidas sem VIH preencheram o protocolo de avaliação no $2 .{ }^{\circ}$ trimestre de gravidez e 44 no pós-parto.

As participantes tinham uma idade média de cerca de 29 anos e uma escolaridade média de 11 anos. No que respeita às variáveis reprodutivas, a gravidez foi planeada por $38,6 \%$ das mulheres seropositivas para o 
VIH e por $84,3 \%$ das mulheres saudáveis. De assinalar ainda que os dois grupos se distinguiam significativamente no que respeita ao planeamento da gravidez $\left[\chi^{2}(1)=21,18 ; p=0,001, V\right.$ de Cramer $=0,47]$. No Quadro 1 , apresentam-se as informações relativas às características sociodemográficas e paridade dos dois grupos.

$\mathrm{Na}$ análise comparativa das grávidas com e sem $\mathrm{VIH}$, verificou-se que os dois grupos eram equivalentes em termos de idade, estado civil e paridade, mas apresentavam diferenças estatisticamente significativas em termos de anos de escolaridade, nível socioeconómico e situação profissional (cf. Quadro I). Como se pode verificar, as grávidas VIH tinham maior probabilidade de ter menos anos de escolaridade ( $d$ de Cohen $=-0,70$ ), estar desempregadas ( $V$ de Cramer $=0,40$ ) e pertencer a um nível socioeconómico mais baixo $(V$ de Cramer $=0,32$ ).

No que respeita às características associadas à infecção porVIH, apresentamos alguns dados adicionais (cf. Quadro II). Nas grávidas VIH a principal circunstância conducente à realização do diagnóstico de infecção por VIH foi a gravidez (actual ou anterior), tendo o diagnóstico ocorrido durante a rotina pré-natal em $51,1 \%$ dos casos. No que se reporta às vias de transmissão, as relações sexuais foram a principal causa de infecção para a maioria das mulheres $(63,8 \%)$. Por fim, em relação ao planeamento da gravidez face ao conhecimento do estado serológico, a maioria não planeou a gravidez, ainda que $17,8 \%$ das mulheres tenha planeado a gravidez conhecendo o seu estado serológico de seropositividade.

\section{Adaptação individual à gravidez}

Os valores médios nos indicadores de adaptação avaliados no primeiro momento de avaliação constam do Quadro III. Pela análise do Quadro III podemos verificar que as grávidas VIH obtêm, em todos os indicadores onde se registaram efeitos estatisticamente significativos, resultados superiores aos das grávidas saudáveis. No que se prende com o planeamento da gravidez, as mulheres que não planearam a gravidez apresentam, em termos gerais, resultados mais elevados nos indicadores de natureza negativa, sendo que apenas no

\begin{tabular}{|c|c|c|c|}
\hline \multicolumn{4}{|c|}{$\begin{array}{l}\text { QUADRO I. Caracterização sociodemográfica e reprodutiva da } \\
\text { amostra }\end{array}$} \\
\hline & $\begin{array}{l}\text { Grávidas VIH } \\
\qquad(n=47)\end{array}$ & $\begin{array}{l}\text { Grávidas sem } \\
\text { VIH }(n=51)\end{array}$ & \multirow[t]{2}{*}{$p$} \\
\hline & $\bar{x}(d p)$ & $\bar{x}(d p)$ & \\
\hline Idade & $28,74(6,01)$ & $29,53(4,06)$ & 0,477 \\
\hline \multirow[t]{2}{*}{ Educação } & $10,17(3,80)$ & $12,61(3,19)$ & 0,001 \\
\hline & $n(\%)$ & $n(\%)$ & \\
\hline \multicolumn{4}{|l|}{ Estado civil } \\
\hline Solteira & $7(15,2)$ & $2(3,9)$ & 0,073 \\
\hline Casada/União de facto & $36(78,3)$ & $48(94,1)$ & \\
\hline Separada/Divorciada & $3(6,5)$ & $1(2,0)$ & \\
\hline \multicolumn{4}{|l|}{ Nível socioeconómico } \\
\hline Baixo & $35(74,5)$ & $22(43,1)$ & 0,007 \\
\hline Médio/Elevado & $12(25,5)$ & $29(56,9)$ & \\
\hline \multicolumn{4}{|l|}{ Situação profissional } \\
\hline Empregada & $23(48,9)$ & $44(86,3)$ & 0,001 \\
\hline Desempregada & $17(36,2)$ & $6(11,7)$ & \\
\hline Doméstica & $5(10,6)$ & - & \\
\hline Estudante & $2(4,3)$ & $1(2,0)$ & \\
\hline \multicolumn{4}{|l|}{ Paridade } \\
\hline Primípara & $25(53,2)$ & $30(58,8)$ & 0,575 \\
\hline Multípara & $22(46,8)$ & $21(41,2)$ & \\
\hline
\end{tabular}

VIH - vírus da imunodeficiência humana

domínio de qualidade de vida «Relações sociais» se observou um efeito estatisticamente significativo, tendo sido marginalmente significativo relativamente à emoção «Tristeza» $(p=0,051)$. Nesta emoção, a média foi superior entre as grávidas seropositivas para o VIH $[M=$ $35,71$ versus $M=23,04]$. Em relação às dimensões de sintomatologia psicopatológica, nenhum efeito se mostrou estatisticamente significativo, com excepção do efeito grupo no Índice de Sintomas Positivos, $F(1,89)$ $=4,91 ; p=0,029$. Neste índice, a média foi mais elevada entre as mulheres infectadas por VIH [ $M=1,67$ versus $M=1,41]$.

Atendendo aos resultados expressos no Quadro III, podemos observar a ausência de efeitos de interacção significativos na maioria dos indicadores, o que permite constatar que o padrão de associação destes indicadores com o planeamento da gravidez é semelhante para as grávidas infectadas pelo VIH e para as grávidas sem 
QUADRO II. Caracterização das variáveis associadas à infecção por vírus da imunodeficiência humana

Grávidas VIH $(n=47)$ $n(\%)$

Momento do diagnóstico

Actual gravidez

$22(46,8)$

Gravidez anterior

Rotina médica

$15(31,9)$

Iniciativa própria

$5(10,6)$

Outra

$3(6,4)$

Categoria de transmissão

Sexual

$30(63,8)$

Drogas injectáveis

$8(17,0)$

Transfusão

Desconhecida

$8(17,0)$

\section{Planeamento de gravidez e}

conhecimento do estado serológico

Planeada, com conhecimento

$8(17,8)$

Planeada, sem conhecimento

$8(17,8)$

Não planeada, com conhecimento

$13(35,6)$

Não planeada, sem conhecimento

$16(28,9)$

VIH - vírus da imunodeficiência humana

condições médicas associadas. Porém, podemos verificar a existência um efeito de interacção estatisticamente significativo para a emoção «Surpresa» $[F(1,89)$ $=4,16 ; p=0,044]$. Concretamente, as grávidas sem VIH que não planearam a gravidez pontuam nesta emoção mais do que as que a planearam, padrão que não se observou nas grávidas VIH, já que uma maior pontuação nesta emoção se registou entre as mulheres que planearam a gravidez.

\section{Adaptação individual ao pós-parto}

Relativamente ao grupo de grávidas infectadas pelo VIH, foram encontradas duas diferenças estatisticamente significativas: para a dimensão psicopatológica «Depressão» $(p=0,010)$ e para a emoção «Tristeza», $(p$ $=0,030$ ). Concretamente, as mulheres seropositivas para o VIH que não planearam a gravidez encontra- vam-se mais tristes e deprimidas nos dias subsequentes ao parto. Em relação às grávidas sem VIH não se registaram diferenças estatisticamente significativas, com excepção da emoção "Culpa», mais elevada entre as mulheres que não planearam a gravidez ( $p=0,045)$.

Adicionalmente a estes resultados, procurámos verificar a existência de associação entre o planeamento da gravidez e a adaptação ao nascimento do bebé. Globalmente, os resultados mostram que o não planeamento da gravidez se associa a maior sintomatologia psicopatológica, maior reactividade negativa e menor percepção de qualidade de vida (cf. Quadro IV).

\section{DISCUSSÃO}

No presente estudo procurámos verificar a associação entre o planeamento de gravidez e a adaptação à gravidez e ao nascimento de um filho em grávidas seropositivas para o VIH comparativamente com grávidas sem patologia médica associada.

Globalmente, os nossos resultados não nos permitiram confirmar a hipótese de que uma gravidez não planeada influencia negativamente a adaptação materna à gravidez. Com efeito, em relação a esta variável, foi apenas encontrado um efeito deste factor, no domínio de qualidade de vida "Relações sociais». De forma geral, as mulheres cuja gravidez foi planeada (de ambos os grupos de estudo) reportam melhores índices de adaptação, ainda que nas grávidas VIH os resultados não se tenham mostrado tão consistentes. No entanto, a inexistência de efeitos significativos de interacção entre as variáveis grupo e planeamento de gravidez mostra que o padrão de associações entre o planeamento da gravidez e os indicadores de adaptação é independente da presença de infecção porVIH. De assinalar, no entanto, que no total dos indicadores, na reactividade emocional foi registado um efeito de interacção entre o planeamento de gravidez e a existência de infecção VIH. Em particular, as grávidas seropositivas para o VIH que planearam a gravidez apresentam valores mais elevados na emoção «Surpresa», ainda que a diferença apenas tenha sido marginalmente significativa. Numa tentativa de melhor compreender estes resultados, análises adicionais mostraram que este resultado superior na emoção «Surpresa» se deve ao momento do diagnóstico. Com efeito, maior «Surpresa» registou-se entre as grávidas cujo diagnóstico ocorreu durante a ac- 


\begin{tabular}{|c|c|c|c|c|c|c|c|}
\hline & \multicolumn{2}{|c|}{ Gravidez planeada } & \multicolumn{2}{|c|}{ Gravidez não planeada } & \multirow{3}{*}{$\begin{array}{c}\text { Grupo } \\
p\end{array}$} & \multirow{3}{*}{\begin{tabular}{|c|} 
Planeamento \\
$P$
\end{tabular}} & \multirow{3}{*}{$\begin{array}{c}\begin{array}{c}\text { Grupo } \mathrm{x} \\
\text { Planeamento }\end{array} \\
P\end{array}$} \\
\hline & \multirow{2}{*}{$\begin{array}{c}\text { com VIH } \\
\overline{\mathrm{x}}(d p)\end{array}$} & \multirow{2}{*}{$\begin{array}{c}\text { sem VIH } \\
\bar{x}(d p)\end{array}$} & \multirow{2}{*}{$\begin{array}{l}\text { com VIH } \\
\overline{\mathrm{x}}(d p)\end{array}$} & \multirow{2}{*}{$\begin{array}{c}\text { sem VIH } \\
\overline{\mathrm{x}}(d p)\end{array}$} & & & \\
\hline & & & & & & & \\
\hline \multicolumn{8}{|c|}{ Reactividade emocional } \\
\hline - Felicidade & $61,42(33,94)$ & $66,60(22,06)$ & $52,15(27,96)$ & $55,71(13,11)$ & 0,516 & 0,136 & 0,904 \\
\hline - Ansiedade & $44,42(23,28)$ & $34,71(20,59)$ & $51,68(23,98)$ & $33,21(26,32)$ & 0,018 & 0,624 & 0,457 \\
\hline - Tristeza & $30,03(35,09)$ & $16,04(16,17)$ & $38,13(29,69)$ & $33,28(17,03)$ & 0,144 & 0,051 & 0,476 \\
\hline - Medo & $38,23(28,42)$ & $14,11(12,72)$ & $32,91(23,20)$ & $24,04(18,89)$ & 0,002 & 0,656 & 0,143 \\
\hline - Surpresa & $35,64(26,10)$ & $16,61(11,07)$ & $28,54(23,82)$ & $29,48(16,32)$ & 0,068 & 0,558 & 0,044 \\
\hline - Culpa & $27,59(22,69)$ & $15,91(11,09)$ & $25,56(20,25)$ & $21,71(12,42)$ & 0,058 & 0,580 & 0,430 \\
\hline - Cólera & $25,16(29,61)$ & $14,32(13,19)$ & $26,35(25,38)$ & $15,19(16,91)$ & 0,046 & 0,850 & 0,977 \\
\hline \multicolumn{8}{|l|}{ Qualidade de vida } \\
\hline - Físico & $54,22(9,01)$ & $55,80(9,56)$ & $55,35(11,22)$ & $55,10(12,35)$ & 0,818 & 0,940 & 0,750 \\
\hline - Psicológico & $65,53(15,38)$ & $70,52(9,59)$ & $71,09(17,84)$ & $70,24(8,82)$ & 0,566 & 0,464 & 0,417 \\
\hline - Relações sociais & $73,48(8,99)$ & $78,33(11,91)$ & $59,90(14,97)$ & $69,05(20,81)$ & 0,067 & 0,003 & 0,569 \\
\hline - Ambiente & $67,05(14,55)$ & $68,53(12,65)$ & $61,33(15,72)$ & $63,39(10,16)$ & 0,553 & 0,125 & 0,956 \\
\hline - Faceta geral & $61,36(21,98)$ & $75,94(11,80)$ & $50,00(19,36)$ & $82,14(15,91)$ & $<0,001$ & 0,566 & 0,053 \\
\hline
\end{tabular}

VIH - vírus da imunodeficiência humana

tual gravidez, comparativamente quer ao grupo de grávidas com diagnóstico anterior, quer ao grupo de grávidas sem VIH. Nenhuma destas diferenças se mostrou estatisticamente significativa, ainda que em comparação com as grávidas em VIH a diferença tenha sido marginalmente significativa.

No segundo momento de avaliação, entre as mulheres infectadas por VIH, foram encontradas diferenças na dimensão «Depressão» e na emoção «Tristeza», que se revelaram mais elevadas entre as mulheres infectadas pelo VIH que não planearam a gravidez. Este resultado está de acordo com o reportado recentemente por Loutfy et al. ${ }^{24}$ que verificaram menores valores na «Felicidade» quando a gravidez não foi planeada. Ainda de acordo com a hipótese colocada, as correlações encontradas em relação aos resultados de adaptação no pós-parto revelam que o não planeamento da gravidez se encontra associado a maior sintomatologia psicopatológica, menor percepção de qualidade de vida e, de forma mais acentuada, a maior reactividade emocional negativa, em particular «Ansiedade», «Culpa» e «Tristeza». Estes resultados estão globalmente em consonância com os resultados apontados na meta-análise de Beck ${ }^{13} \mathrm{e}$ nos estudos de Cheng et al. ${ }^{14} \mathrm{e}$ Najman, Morrison, Williams, Andersen e Keeping, ${ }^{12}$ nomeadamente de que o não planeamento da gravidez se encontra significativamente associado a maior probabilidade de depressão pós-parto. São ainda parcialmente consistentes com o estudo de Schawrz et al., ${ }^{15}$ que registaram menor qualidade de vida entre as mulheres que não planearam a gravidez. São, no entanto, distintos de um estudo conduzido no Canadá, ${ }^{16}$ que não demonstrou qualquer associação entre gravidez não planeada e distress psicológico materno. Esta diferença poderá estar relacionada com diferenças culturais da nossa amostra, mas também pelas opções metodológicas dos autores, que analisaram a associação entre estas variáveis em função dos grupos etários (idade inferior e superior a 


\begin{tabular}{lc} 
QUADRO IV. Correlação entre a existência de planea- \\
mento da gravidez e os indicadores de adaptação \\
$\begin{array}{l}\text { Variáveis } \\
\text { Sintomatologia psicopatológica }\end{array}$ \\
Somatização & 0,797 \\
Obsessões-Compulsões & 0,629 \\
Sensibilidade interpessoal & 0,036 \\
Depressão & 0,005 \\
Ansiedade & 0,920 \\
Hostilidade & 0,102 \\
Ansiedade fóbica & 0,542 \\
Ideação paranóide & 0,098 \\
Psicoticismo & 0,036 \\
\hline Reactividade emocional & \\
Felicidade & 0,176 \\
Ansiedade & 0,001 \\
Medo & 0,017 \\
Surpresa & 0,006 \\
Culpa & 0,057 \\
Cólera & 0,001 \\
Relisteza & 0,006 \\
\hline Ambicota genões sociais & 0,002 \\
\hline
\end{tabular}

\section{0 anos)}

No cômputo geral, estes dados corroboram a literatura científica que sublinha que uma gravidez planeada, ao contrário de uma gravidez não planeada, pressupõe uma motivação e uma decisão prévia pela maternidade. ${ }^{32}$ Donde, o desejo de querer ter um filho pode conduzir a uma melhor adaptação às mudanças que este período tem subjacente. Por outro lado, tendo em consideração que o nascimento de um filho está associado a mudanças de variadas naturezas e, como mudança que representa, pode implicar stresse, ${ }^{33}$ os dados obtidos podem de igual forma significar um maior con- fronto com os desafios inerentes a esse mesmo nascimento, nomeadamente pelas mudanças, reorganizações (por exemplo, a nível individual, do casal e familiar) e responsabilidades que acarretam.

O presente estudo adiciona à literatura existente a importância de considerar os determinantes reprodutivos na adaptação na transição para a maternidade, em particular o planeamento da gravidez. No entanto, não se encontra isento de limitações. Em primeiro lugar, as limitações impostas pela amostragem por conveniência devem ser consideradas. Este procedimento pode implicar um viés na selecção da amostra, ainda que a consulta da Maternidade Doutor Daniel de Matos receba utentes de diversos pontos do país, embora predominantemente da região Centro. Neste sentido, tal procedimento não permite assegurar a representatividade da amostra, limitando, por conseguinte, a generalização dos resultados. Uma segunda limitação diz respeito ao tamanho da amostra, que limita as conclusões do presente estudo, mas também o poder para detectar diferenças (efeitos pequenos). Adicionalmente, a baixa taxa de resposta na avaliação após o parto, em particular nas grávidas VIH, e apesar dos nossos esforços, é uma importante limitação a ter em consideração. Deste modo, grupos de maiores dimensões (e mais semelhantes em termos de características sociodemográficas) poderiam fornecer informações mais adequadas sobre a adaptação na transição para a maternidade em circunstâncias diferentes, isto é, em contextos normativos e contextos de risco, de forma a melhor compreender, separadamente, as trajectórias de adaptação ao longo da gravidez.

Uma outra limitação a referir é o facto de ter sido usado um número considerável de testes estatísticos sem terem sido definidas previamente variáveis principais e secundárias nem ter sido feita qualquer correcção para os múltiplos testes realizados. A realização de múltiplos testes, aumentando a probabilidade de um ou mais dos resultados ser devido ao acaso, deixa de nos permitir interpretar valores de $p$ inferiores a 0,05 como necessariamente significativos.

Dados os objectivos centrais do presente estudo, não podemos deixar de responder à inevitável questão sobre as suas implicações práticas (clínicas) dos resultados obtidos, bem como alertar para a premência que o planeamento familiar e a vigilância pré-natal podem as- 
sumir no contexto particular da infecção por VIH. Esta vigilância tem sido entendida como um componente insubstituível da saúde materna e do desenvolvimento do bebé e é uma das áreas de intervenção prioritária do Plano Nacional de Saúde. ${ }^{34}$ Neste contexto, é ainda de grande importância que não se ignorem as mulheres não grávidas. Como advogam o American College of Obstetrics and Gynecology e o Institute of Medicine, todas as mulheres em idade reprodutiva devem receber aconselhamento VIH pré-concepção e a todas as grávidas deve ser oferecido o teste VIH como parte integrante da rotina médica primária, independentemente dos factores de risco e da taxa de prevalência na comunidade. Nesta linha, os planos de gravidez e maternidade devem também ser discutidos também com as mulheres não-grávidas infectadas, para que estas possam tomar decisões informadas e ponderadas.

Estudos futuros, com amostras de maior dimensão e com mais momentos de avaliação (por exemplo, até seis meses após o parto) poderão contribuir para uma compreensão mais clara do processo de adaptação na transição para a maternidade subjacente a uma gravidez não planeada. Por fim, entendemos também que outras variáveis poderiam ser consideradas neste estudo (e.g., gravidezes não planeadas anteriores; história de interrupções; número de filhos; planeamento individual ou do casal; condições socioeconómicas, etc.). Partindo da noção de que os efeitos de uma gravidez não planeada podem variar consideravelmente entre as mulheres, uma consideração ecológica, isto é, de diferentes contextos de influência, poderá permitir uma melhor investigação sobre esses mesmos efeitos em termos de ajustamento emocional e, adicionalmente, providenciar medidas mais fiáveis e válidas, que possam ser utilizadas para reforçar políticas e reforçar programas de planeamento familiar.

\section{REFERÊNCIAS BIBLIOGRÁFICAS}

1. Canavarro MC. Gravidez e maternidade: representações e tarefas de desenvolvimento. In: Canavarro MC, editor. Psicologia da Gravidez e da Maternidade. Coimbra: Quarteto Editora; 2001. p. 17-49.

2. Cox MJ, Paley B, Burchinal M, Payne CC. Marital perceptions and interactions and the transition to parenthood. J Marriage Fam 1999 Aug; 61 (3): 611-25.

3. Joyce T, Kaestner R, Korenman S. The stability of pregnancy intentions and pregnancy-related maternal behaviors. Mater Child Health J 2000 Sep; 4 (3): 171-8.
4. Barrett $G$, Wellings $K$. What is a 'planned' pregnancy? Empirical data from a Bristish study. Soc Sci Med 2002 Aug; 55 (4): 545-57.

5. Brown SS, Eisenberg L. The best intentions: unintended pregnancies and the well-being of children and families. Washington, DC: National Academy Press; 1995.

6. Kost K, Landry DJ, Darroch JE. The effects of pregnancy planning status on birth outcomes and infant care. Fam Plann Perspect 1998 Sep-Oct; 30 (5): 223-30.

7. Arslan Özkan I, Mete S. Pregnancy planning and antenatal health behaviour: findings from one maternity unit in Turkey. Midwifery 2010 Jun; 26 (3): 338-47.

8. Carvalho P, Loureiro M, Simões MR. Gravidez e risco psicopatológico. Psychologica 2007; 46: 105-24.

9. Barnard KE, Solchany JE. Mothering. In: Handbook of Parenting. MH Bornstein, editors. Mahwah, NJ: Erlbaum Associates; 2002. p. 3-26.

10. Belsky J, Rovine M. Patterns of marital change across the transition to parenthood: Pregnancy to three years postpartum. J Marriage Fam 1990 Feb; 52 (1): 5-19.

11. Feinberg ME. Coparenting and the transition to parenthood: a framework for prevention. Clin Child Fam Psychol Rev 2002 Sep; 5 (3): 173-95.

12. Najman JM, Morrison J, Williams G, Andersen M, Keeping JD. The mental health of women 6 months after they gave birth to an unwanted baby: a longitudinal study. Soc Sci Med 1991; 32 (3): 241-7.

13. Beck CT. Predictors of postpartum depression: an update. Nurs Res 2001 Sep-Oct; 50 (5): 275-85.

14. Cheng D, Schwarz EB, Douglas E, Horon I. Unintended pregnancy and associated maternal preconception, prenatal and postpartum behaviors. Contraception 2009 Mar; 79 (3): 194-8.

15. Schwarz EB, Smith R, Steinauer J, Reeves MF, Caughey AB. Measuring the effects of unintended pregnancy on women's quality of life. Contraception 2008 Sep; 78 (3): 204-10.

16. Maximova K, Quesnel-Vallée A. Mental health consequences of unintended childlessness and unplanned births: gender differences and life course dynamics. Soc Sci Med 2009 Mar; 68 (5): 850-7.

17. Schmiege S, Russo NF. Depression and unwanted first pregnancy: longitudinal cohort study. Br Med J 2005 Dec 3; 331 (7528): 1303-6.

18. Campbell C. Women, Families, \& AIDS: a Sociological Perspective on the Epidemic in America. Cambridge: Cambridge University Press; 1999.

19. Kline A, Strickler J, Kempf J. Factors associated with pregnancy and pregnancy resolution in HIV seropositive women. Soc Sci Med 1995 Jun; 40 (11): 1539-47.

20. Pereira M, Canavarro MC. Determinantes sócio-demográficos e clínicos na decisão reprodutiva em mulheres infectadas pelo VIH/SIDA. Acta Obstet GinecoL Port 2008; 2 (2): 56-64.

21. Sowell RL, Misener TR. Decision to have a baby by HIV-infected women. West J Nurs Res 1997 Feb; 19 (1): 56-70.

22. Sowell RL, Phillips KD, Misener TR. HIV-infected women and motivation to add children to their families. J Fam Nurs 1999 Aug; 5 (3): 316-31.

23. Pereira $M$, Canavarro MC. Vulnerabilidade de género e outras dimensões de influência na adaptação ao VIH/SIDA e à gravidez e maternidade. Análise Psicológica 2007; 3 (XXV): 503-15.

24. Loutfy M, Raboud J, Wong J, Yudin M, Diong C, Blitz S, et al. High prevalence of unintended pregnancies in HIV-positive women of reproductive age in Ontario, Canada: a retrospective study. HIV Med 2012 Feb; 13 (2): 
107-17.

25. Derogatis LR. BSI: Brief Symptom Inventory: Administration, scoring and procedures manual. Minneapolis: Natural Computers System; 1993.

26. Canavarro MC. Inventário de Sintomas Psicopatológicos (BSI): uma revisão crítica dos estudos realizados em Portugal. In: MR Simões, C Machado, M Gonçalves, LAlmeida, coord. Avaliação psicológica: instrumentos validados para a população Portuguesa. Vol. III. Coimbra: Quarteto Editora; 2007. p. 305-31.

27. Carlson CR, Collins FL, Stewart JF, Porzelius J, Nitz JA, Lind CO. The assessment of emotional reactivity: A scale development and validation study. J Psychopath Behav Assess 1989 Dec; 11 (4): 313-25.

28. Moura-Ramos M. Adaptação materna e paterna ao nascimento de um filho: Percursos e contextos de influência [Dissertação de Mestrado] Coimbra: Faculdade de Psicologia e de Ciências da Educação da Universidade de Coimbra; 2006.

29. WHOQOL Group. Development of World Health Organization WHOQOLBREF Quality of Life Assessment. Psychol Med 1998 May: 28 (3): 551-8.

30. Vaz Serra A, Canavarro MC, Simões MR, Pereira M, Gameiro S, Quarti-lho $\mathrm{MJ}$, et al. Estudos psicométricos do instrumento de avaliação da qualidade de vida da Organização Mundial de Saúde (WHOQOL-Bref) para Português de Portugal. Psiquiatria Clínica 2006; 27 (1): 41-9.

31. Cohen J.A power prime. Psychol Bull 1992 Jul; 112 (1): 155-9.

32. MillerWB. Childbearing motivations, desires, and intentions: $A$ theoretical framework. Genet Soc Gen Psychol Monogr 1994 May; 120 (2): 223-58. 33. Boss P. Family stress management. Beverly Hills: Sage Publications; 1988. 34. Circular Normativa n. ${ }^{\circ}$ 02/DSMIA, de 16 de Janeiro de 2006. Lisboa: Ministério da Saúde; 2006.

\section{CONFLITOS DE INTERESSE}

Os autores declaram não ter qualquer tipo de conflito de interesses. O presente estudo foi desenvolvido no âmbito do Grupo de Investigação "Relações, Desenvolvimento e Saúde", da Unidade I\&D Instituto de Psicologia Cognitiva, Desenvolvimento Vocacional e Social (FEDER/POCTI-SFA160-192). O estudo foi financiado pela Coordenação Nacional para a Infecção VIH/sida (Proc. 11-7.3/2004) e por uma bolsa da Fundação para a Ciência e Tecnologia (SFRH/BD/19126/2004).

\section{ENDEREÇO PARA CORRESPONDÊNCIA}

Marco Pereira

Instituto de Psicologia Cognitiva, Desenvolvimento Vocacional e Social

Faculdade de Psicologia e de Ciências da Educação da Universidade de Coimbra

Rua do Colégio Novo, Apartado 6153, 3001-802 Coimbra, Portugal

E-mail: marcopereira@fpce.uc.pt

Recebido em 25/11/2011

Aceite para publicação em 04/04/2012

\section{ABSTRACT}

\section{PREGNANCY PLANNING AND ADAPTATION TO MOTHERHOOD AMONG HIV-POSITIVE PREGNANT WOMEN}

Objectives: To analyse the influence of the planning of pregnancy on the adaptation to motherhood among HIV-infected women compared to healthy pregnant women.

Design: Observational study with assessments performed during the second trimester of pregnancy and 2 to 4 days postpartum.

Population: Ninety-eight women including 47 HIV-positive pregnant women and 51 healthy pregnant women

Setting: Hospitais da Universidade de Coimbra: Área de Gestão Integrada de Saúde Materno-Fetal -Psychological Intervention Unit of of the Doctor Daniel de Matos Maternity Hospital and the Doctor Alfredo da Costa Maternity Hospital (Lisbon).

Methods: The adaptation to motherhood was assessed by the administration of three self-report questionnaires designed to assess psychopathological symptoms (Brief Symptom Inventory), emotional reactivity (Emotional Assessment Scale), and quality of life (WHOQOL-Bref). The association between the planning of pregnancy and adaptation to motherhood was analysed comparing HIV-positive pregnant women and healthy pregnant women. Inferential statistics were used with a significance level of 0.05 .

Results: The results support our hypothesis that unplanned pregnancies are associated with worse adaptation in the transition to motherhood. This is greater among HIV-infected women. In the postpartum period, unplanned pregnancies were significantly associated with higher psychopathology, higher negative emotional reactivity, and poorer quality of life.

Conclusions: Our findings highlight the importance the planning of pregnancy in the adjustment to pregnancy and the postpartum period. These findings also stress the importance of the discussion of childbearing plans with HIV-infected women before pregnancy.

Keywords: Family Planning; Adaptation, Psychological; Human immunodeficiency virus 